\title{
THyMe+: Temporal Hypergraph Motifs and Fast Algorithms for Exact Counting
}

\author{
Geon Lee \\ Graduate School of AI, KAIST \\ geonlee0325@kaist.ac.kr
}

\author{
Kijung Shin \\ Graduate School of AI and School of Electrical Engineering, KAIST \\ kijungs@kaist.ac.kr
}

\begin{abstract}
Group interactions arise in our daily lives (email communications, on-demand ride sharing, comment interactions on online communities, to name a few), and they together form hypergraphs that evolve over time. Given such temporal hypergraphs, how can we describe their underlying design principles? If their sizes and time spans are considerably different, how can we compare their structural and temporal characteristics?

In this work, we define 96 temporal hypergraph motifs (THmotifs), and propose the relative occurrences of their instances as an answer to the above questions. TH-motifs categorize the relational and temporal dynamics among three connected hyperedges that appear within a short time. For scalable analysis, we develop $\mathrm{THYME}^{+}$, a fast and exact algorithm for counting the instances of TH-motifs in massive hypergraphs, and show that THYME $^{+}$is at most $2,163 \times$ faster while requiring less space than baseline. Using it, we investigate 11 real-world temporal hypergraphs from various domains. We demonstrate that $\mathbf{T H}$ motifs provide important information useful for downstream tasks and reveal interesting patterns, including the striking similarity between temporal hypergraphs from the same domain.
\end{abstract}

\section{INTRODUCTION}

Interactions in real-world systems are complex, and in many cases, they are beyond pairwise: email communications, ondemand ride sharing, comment interactions on online communities, to name a few. These group interactions together form a hypergraph, which consists of a set of nodes and a set of hyperedges (see Fig. 11a) for an example). Each hyperedge is a subset of any number of nodes, and by naturally representing a group interaction among multiple individuals or objects, it contributes to the powerful expressiveness of hypergraphs.

Recently, several empirical studies have revealed structural and temporal properties of real-world hypergraphs. Pervasive structural patterns include (a) heavy-tailed distributions of degrees, edge sizes, and intersection sizes [1]; (b) giant connected components [2], and small diameters [2]; and (c) substantial overlaps of hyperedges with homophily [3]. Temporal properties observed commonly in various time-evolving hypergraphs include (a) significant overlaps between temporally adjacent hyperedges [4]; and (b) diminishing overlaps, densification, and shrinking diameters [1].

In addition to these macroscopic properties, local connectivity and dynamics in real-world hypergraphs have been studied. Benson et al. [5] examined the interactions among a fixed number of nodes, with a focus on their relations with the emergence of a hyperedge containing all the nodes. Lee et al. [6] inspected the overlaps between three hyperedges, which they categorize into 26 patterns called hypergraph motifs (h-motifs). Comparing the relative counts of each h-motif's

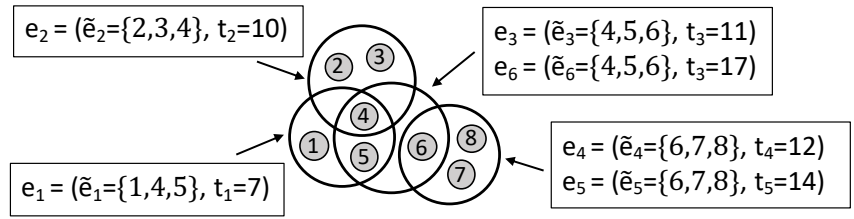

(a) An example temporal hypergraph

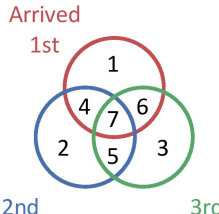

(b) 7 regions for defining TH-motifs

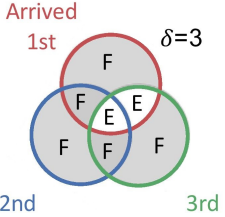

(c) The definition of TH-motif 77

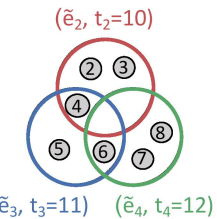

(d) An instance of TH-motif 77
Fig. 1: (a) A temporal hypergraph with 8 nodes and 6 temporal hyperedges. (b) The 7 regions in the Venn diagram representation for defining TH-motifs. (c) The definition of TH-motif 77. 'F' and 'E' stand for 'filled' and 'empty', respectively. (d) The sequence $\left\langle e_{2}, e_{3}, e_{4}\right\rangle$ is an instance of TH-motif 77 .

instances revealed that local structures are particularly similar between hypergraphs from the same domain but different across domains. In h-motifs, however, temporal dynamics are completely ignored.

This line of research has also revealed that specialized analysis tools (e.g., h-motifs [6] and multi-level decomposition [2]) are useful for extracting unique high-order information that hypergraphs convey and also for coping with additional complexity due to the flexibility in the size of hyperedges. Simply utilizing graph analysis tools (e.g., network motifs [7]) after converting hypergraphs into pairwise graphs is often limited in addressing the above challenges [6], [8].

Motivated by interesting patterns that temporal network motifs revealed in ordinary graphs [9]-[13], we define 96 temporal hypergraph motifs (TH-motifs) for local pattern analysis of time-evolving hypergrpahs. TH-motifs generalize the notion of static h-motifs, which completely ignore temporal information, and describe both relational and temporal dynamics among three connected hyperedges that arrive within a short time. Specifically, given three connected hyperedges $e_{i}, e_{j}$, and $e_{k}$, all of which arrive within $\delta$ time units, THmotifs describe their connectivity based on the emptiness of the seven subsets of them shown in Fig. 11b). In the temporal perspective, the relative arrival orders of $e_{i}, e_{j}$, and $e_{k}$ are taken into account, and thus patterns that are indistinguishable 
using static h-motifs can be characterized using TH-motifs.

Given a temporal hypergraph, where a timestamp is attached to each hyperedge (see Fig. 1. (a) for an example), we summarize its local structural and temporal characteristics using the relative occurrence of 96 TH-motifs' instances. That is, we obtain a vector of length 96 regardless of the sizes and time spans of hypergraphs, and thus local characteristics of different hypergraphs can easily be compared.

Another focus of this paper is the problem of counting TH-motifs' instances. Since the number of three connected hyperedges can be orders of magnitude larger than the number of hyperedges, directly enumerating all of them is computationally prohibitive, especially for massive hypergraphs. We develop THyME ${ }^{+}$(Temporal Hypergraph Motif Census), which exactly counts each TH-motif's instances while avoiding direct enumeration. In our experiments, $\mathrm{THYME}^{+}$is up to $2,163 \times$ faster than the direct extension of a recent exact temporal network motif counting algorithm [9], which enumerates every static h-motif in the induced static hypergraph. $\mathrm{THYME}^{+}$makes the best use of our two findings in real-world hypergraphs that temporal hyperedges tend to be (1) repetitive and (2) temporally local. These findings about duplicated (i.e., completely overlapped) hyperedges complement the findings in [4], which focus mainly on partial overlaps.

Using TH-motifs and $\mathrm{THYME}^{+}$, we investigate 11 realworld hypergraphs from 5 distinct domains. Our empirical study demonstrates that TH-motifs are informative, capturing both structural and temporal characteristics. Specifically, using the counts of incident TH-motifs' instances as features brings up to $\mathbf{2 5 . 7} \%$ improvement in the accuracy of a hyperedge prediction task, compared to when static h-motifs are used instead of TH-motifs. Moreover, TH-motifs reveal interesting patterns, including the striking similarity between hypergraphs from the same domain.

In summary, our contributions are as follow:

1) New concept: We define 96 temporal hypergraph motifs (TH-motifs) for characterizing local structures and dynamics in hypergraphs of various sizes.

2) Fast and exact algorithms: We develop fast algorithms for exactly counting the instances of TH-motifs, and they are up to $2,163 \times$ faster than baseline.

3) Empirical discoveries: We demonstrate the usefulness of TH-motifs by uncovering the design principles of 11 realworld temporal hypergraphs from 5 different domains.

Reproducibility: The source code and datasets used in this work are available at https://github.com/geonlee0325/THyMe.

In Section II] we review preliminaries and related prior works. In Section III, we present the concept of TH-motifs. In Section IV, we develop algorithms for counting the instances of TH-motifs. In Section $\mathrm{V}$, we empirically analyze real-world temporal hypergraphs through the lens of TH-motifs. Lastly, in Section VI] we offer conclusions.

\section{PRELIminaries AND RELATED WORKS}

In this section, we first review the concept of hypergraphs. Then, we introduce hypergraph motifs (h-motifs), which is de-
TABLE I: Frequently-used notations.

\begin{tabular}{c|l}
\hline Notation & Definition \\
\hline$T=(V, \mathcal{E})$ & temporal hypergraph with temporal hyperedges $\mathcal{E}$ \\
$G_{T}=\left(V, E_{\mathcal{E}}\right)$ & induced static hypergraph of the temporal hypergraph $T$ \\
\hline$e_{i}=\left(\tilde{e}_{i}, t_{i}\right)$ & temporal hyperedge with nodes $\tilde{e}_{i}$ arrived at time $t_{i}$ \\
$I(\tilde{e})$ & set of temporal hyperedges whose nodes are $\tilde{e}$ \\
$h\left(\tilde{e}_{i}, \tilde{e}_{j}, \tilde{e}_{k}\right)$ & TH-motif corresponding to an instance $\left\langle e_{i}, e_{j}, e_{k}\right\rangle$ \\
\hline$P=\left(V_{P}, E_{P}\right)$ & projected graph in THYME \\
$Q=\left(V_{Q}, E_{Q}, t_{Q}\right)$ & projected graph in THYME \\
\hline
\end{tabular}

signed for static hypergraphs. Lastly, we discuss other related works. Refer to Table I for the frequently-used notations.

\section{A. Basic Concepts: Static and Temporal Hypergraphs}

A hypergraph $G=(V, E)$ consists of a set of nodes $V=$ $\left\{v_{1}, \ldots, v_{|V|}\right\}$ and a set of hyperedges $E=\left\{\tilde{e}_{1}, \ldots, \tilde{e}_{|E|}\right\}$. Each hyperedge $\tilde{e} \in E$ is a non-empty set of an arbitrary number of nodes. A temporal hypergraph $T=(V, \mathcal{E})$ on a node set $V$ is an ordered sequence of temporal hyperedges. Each $i^{\text {th }}$ temporal hyperedge $e_{i}=\left(\tilde{e}_{i}, t_{i}\right)$ where $\tilde{e}_{i} \subseteq V$ is the set of nodes and $t_{i}$ is the time of arrival. Two distinct temporal hyperedges $e_{i}=$ $\left(\tilde{e}_{i}, t_{i}\right)$ and $e_{j}=\left(\tilde{e}_{j}, t_{j}\right)$ are duplicated if they share exactly same set of nodes, i.e., $\tilde{e}_{i}=\tilde{e}_{j}$. We assume the sequence is ordered and timestamps are unique, i.e., if $i<j$, then $t_{i}<$ $t_{j}$. We denote the set of temporal hyperedges whose nodes are $\tilde{e}$ (i.e., those inducing $\tilde{e}$ ) by $I(\tilde{e}):=\left\{e_{i}=\left(\tilde{e}_{i}, t_{i}\right) \in\right.$ $\left.\mathcal{E}: \tilde{e}_{i}=\tilde{e}\right\}$. The temporal hypergraph $T$ induces a static hypergraph $G_{T}=\left(V, E_{\mathcal{E}}\right)$ where timestamps and duplicated temporal hyperedges are ignored. That is, a hyperedge $\tilde{e} \in E_{\mathcal{E}}$ in $G_{T}$ exists if and only if $I(\tilde{e}) \neq \emptyset$. Notably, the number of temporal hyperedges is typically much larger than that of static hyperedges in the induced hypergraph, i.e., $|\mathcal{E}| \gg\left|E_{\mathcal{E}}\right|$.

\section{B. Static Hypergraph Motifs (h-motifs)}

Hypergraph motifs (h-motifs) [6] are tools for understanding the local structural properties of static hypergraphs. Given three connected hyperedges, h-motifs describe their connectivity patterns by the emptiness of each of seven subsets: (1) $\tilde{e}_{i} \backslash \tilde{e}_{j} \backslash \tilde{e}_{k}$, (2) $\tilde{e}_{j} \backslash \tilde{e}_{k} \backslash \tilde{e}_{i}$, (3) $\tilde{e}_{k} \backslash \tilde{e}_{i} \backslash \tilde{e}_{j}$, (4) $\tilde{e}_{i} \cap \tilde{e}_{j} \backslash \tilde{e}_{k}$, (5) $\tilde{e}_{j} \cap \tilde{e}_{k} \backslash \tilde{e}_{i}$, (6) $\tilde{e}_{k} \cap \tilde{e}_{i} \backslash \tilde{e}_{j}$, and (7) $\tilde{e}_{i} \cap \tilde{e}_{j} \cap \tilde{e}_{k}$. While there can exist $2^{7}$ possible cases of emptiness, 26 cases of them are considered after excluding symmetric, duplicated, and disconnected ones. Since non-pairwise interactions among the hyperedges (such as $\tilde{e}_{i} \cap \tilde{e}_{j} \cap \tilde{e}_{k}$ ) are taken into account, h-motifs effectively captures the high-order information of the overlapping patterns of the hyperedges. It is shown empirically that their occurrences in the real-world hypergraphs are significantly different from those in randomized hypergraphs. Moreover, the relative occurrences are particularly similar between hypergraphs from the same domain, while they are distinct between hypergraphs from different domains. Note that $h-$ motifs, which is originally designed for static hypergraphs, completely ignore temporal information.

\section{Other Related Works}

In this subsection, we review prior works on network motifs and empirical analysis of hypergraphs. 


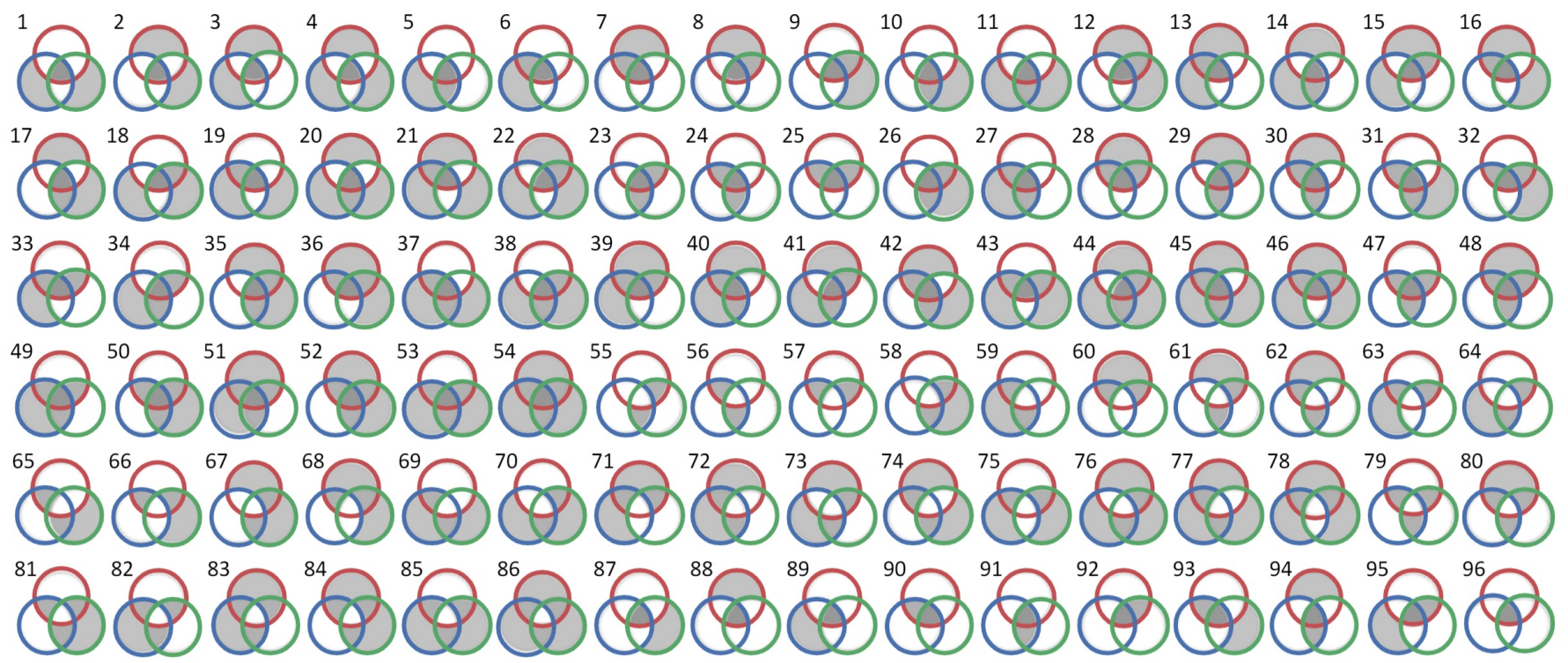

Fig. 2: The 96 temporal hypergraph motifs (TH-motifs). In each TH-motif, the red hyperedge arrives first followed by the blue one and then the green one. Each of the 7 distinct regions in the Venn diagram representation is colored white if it is empty, and it is colored grey if it is filled with at least one node. See Fig. 11(d) for an instance of TH-motif 77.

Network Motifs. Network motifs are fundamental building blocks of real-world graphs [7], [14]. Their relative occurrences in real-world graphs are significantly different from those in randomized ones [7] and unique within each domain [15]. While they were originally defined on a static graph, they have been extended to temporal [9], heterogeneous [10], [16], and bipartite [17] graphs, as well as hypergraphs [6]. Their usefulness has been demonstrated in a wide range of graph applications including community detection [18]-[22], ranking [23], and embedding [24]-[28].

Temporal Network Motifs: The notion of network motifs has been extended to temporal networks to describe patterns in sequences of temporal edges. Several definitions of temporal motifs have been used, and most of them consider the temporal connectivity between the edges. In [11] and [12], they consider $\delta$-adjacency between temporal edges. That is, every consecutive edges should share a node and arrive within in $\delta$ time units. Several counting algorithms for such patterns have been proposed [11]-[13]. Another definition of temporal motifs describes patterns of sequences of temporal edges where all edges arrive within $\delta$ time units [9] while taking their relative arrival orders into consideration. In this work, we define THmotifs based on the notion of temporal motifs defined in [9] due to its simplicity and effectiveness.

Empirical Analysis of Real-world Hypergraphs: Empirical analysis of global [2], [3] and local [5], [6] structural patterns and temporal patterns [1], [4], [5] of real-world hypergraphs has been performed, as discussed in detail in Section [1]

\section{Proposed Concepts}

In this section, we propose temporal hypergraph motifs (THmotifs), which are tools for understanding the local structural and temporal characteristics of temporal hypergraphs. We introduce the definition and their relevant concepts.
Definition: TH-motifs describe structural and temporal patterns in sequences of three connected temporal hyperedges that are close in time. Note that three hyperedges are connected if and only if one among them overlaps with the others. Specifically, given three connected temporal hyperedges $\left\langle e_{i}=\right.$ $\left.\left(\tilde{e}_{i}, t_{i}\right), e_{j}=\left(\tilde{e}_{j}, t_{j}\right), e_{k}=\left(\tilde{e}_{k}, t_{k}\right)\right\rangle$ where $t_{i}<t_{j}<t_{k}$ and $t_{k}-t_{i} \leq \delta$ (i.e., they arrive within a predefined time interval $\delta$ ), TH-motifs describe the emptiness of the 7 subsets: (1) $\tilde{e}_{i} \backslash \tilde{e}_{j} \backslash \tilde{e}_{k}$, (2) $\tilde{e}_{j} \backslash \tilde{e}_{k} \backslash \tilde{e}_{i}$, (3) $\tilde{e}_{k} \backslash \tilde{e}_{i} \backslash \tilde{e}_{j}$, (4) $\tilde{e}_{i} \cap \tilde{e}_{j} \backslash \tilde{e}_{k}$, (5) $\tilde{e}_{j} \cap \tilde{e}_{k} \backslash \tilde{e}_{i}$, (6) $\tilde{e}_{k} \cap \tilde{e}_{i} \backslash \tilde{e}_{j}$, and (7) $\tilde{e}_{i} \cap \tilde{e}_{j} \cap \tilde{e}_{k}$. That is, in the structural aspect, TH-motif describes the emptiness of the seven distinct regions in the Venn diagram representation (see Fig. 1.b)), effectively capturing the high-order connectivity among three hyperedges. In the temporal aspects, TH-motifs take the relative arrival orders of three hyperedges and their time interval into consideration. While there can exist $2^{7}$ possible cases of emptiness, we consider 96 cases of them, which are called TH-motif 1 to TH-motif 96, after excluding those describing disconnected hyperedges. We visualize the $96 \mathrm{TH}-$ motifs in Fig. 2. Recall that static h-motifs completely ignore temporal information, and also assume that every hyperedge is unique, while TH-motifs also describe the patterns among duplicated temporal hyperedges. Thus, while static h-motifs distinguish only 26 different patterns, TH-motifs distinguish 96 different patterns by considering temporal dynamics in addition to connectivity.

Instance of TH-motifs: A sequence $\left\langle e_{i}, e_{j}, e_{k}\right\rangle$ of three temporal hyperedges is an instance of TH-motif $t$ if their relational and temporal dynamics are described by TH-motif $t$ (see Fig. 11d) for an example). For each instance $\left\langle e_{i}, e_{j}, e_{k}\right\rangle$, we denote its corresponding TH-motif by $h\left(\tilde{e}_{i}, \tilde{e}_{j}, \tilde{e}_{k}\right)$.

Triple, Pair, and Single Inducing TH-motifs: The 96 THmotifs can be categorized into three types based on the number of underlying static hyperedges. A TH-motif is triple-inducing 


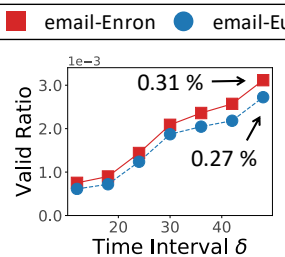

(a) email

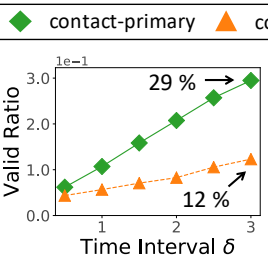

(b) contact

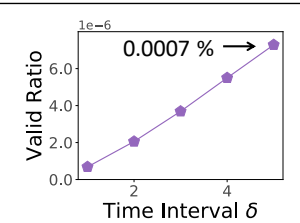

(c) threads
Fig. 3: Only a small fraction of static h-motifs' instances in the induced static hypergraphs are induced by any valid instance of TH-motifs. Results in small datasets where the instances of static h-motifs can be exactly counted are reported.

if underlying hyperedges in its instance $\left\langle e_{i}, e_{j}, e_{k}\right\rangle$ are distinct (i.e., $\tilde{e}_{i} \neq \tilde{e}_{j}, \tilde{e}_{j} \neq \tilde{e}_{k}$, and $\tilde{e}_{k} \neq \tilde{e}_{i}$ ), as in TH-motifs 1-86. If two are duplicated while the remaining one is different, as in TH-motifs $87-95$, it is pair-inducing. If all three hyperedges are duplicated, as in TH-motif 96, it is single-inducing.

\section{Counting Algorithms}

In this section, we describe methodologies for exactly counting the instances of each TH-motifs in the input temporal hypergraph. We first present DP, which extends a recent exact counting algorithm [9] for temporal network motifs. Then, we describe THYME, a preliminary version of our proposed algorithm $\mathrm{THYME}^{+}$. Lastly, we propose $\mathrm{THYME}^{+}$(Temporal Hypergraph Motif Census), a fast and efficient algorithm that addresses the limitations of the previous ones.

Remarks: The problem of counting TH-motifs has additional technical challenges while it bears some similarity with counting static h-motifs or temporal network motifs. First, the number of temporal hyperedges is typically much larger than that of hyperedges in the underlying static hypergraph. For example, the 11 considered real-world temporal hypergraphs (see Section V-A) have up to $1.2-22.0 \times$ more hyperedges than the underlying static ones. This incurs significant bottlenecks of enumeration methods, and thus fast algorithms are demanded. Temporal network motifs are defined only by pairwise interactions among a fixed number of nodes and their timestamps. However, TH-motifs are defined not just by pairwise interactions but also by non-pairwise interactions among three hyperedges, in addition to their timestamps.

\section{A. Dynamic Programming (DP): Extension of [9]}

We present Dynamic Programming (DP), which is a baseline approach for counting the instances of each TH-motif in the input temporal hypergraph $T$.

Counting DP: Given an input temporal hypergraph $T=$ $(V, \mathcal{E})$, DP enumerates the instances of static h-motifs in the induced static hypergraph $G_{T}=\left(V, E_{\mathcal{E}}\right)$. This step can be processed by using an existing algorithm provided in [6]. For each instance $\left\{\tilde{e}_{i}, \tilde{e}_{j}, \tilde{e}_{k}\right\}$ of static h-motif in $G_{T}$, DP counts the instances of each TH-motifs whose temporal hyperedges (a) induce the static h-motif instance and (b) arrive within $\delta$ time. To this end, we adapt the dynamic programming scheme provided by [9], as described in detail in Appendix A.

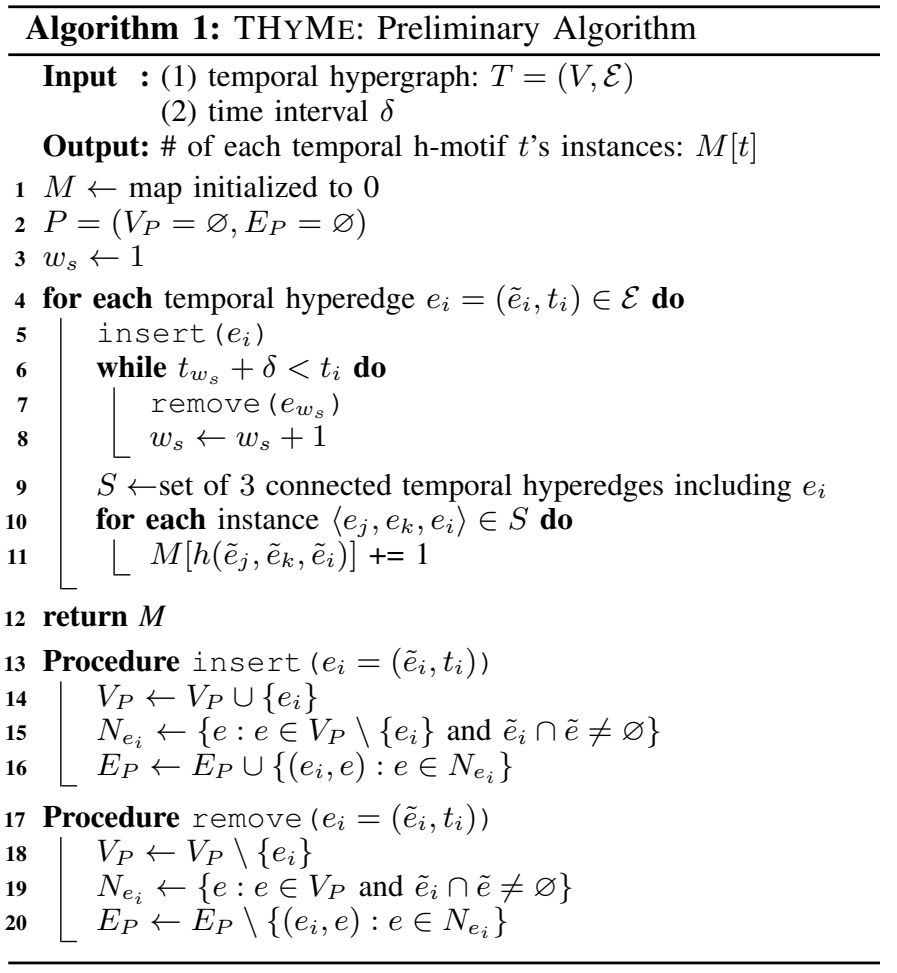

Limitations of DP: Using dynamic programming, DP avoids enumerating over all instances of TH-motifs. However, it still enumerates all instances of static h-motifs in the induced hypergraph $G_{T}$, most of which however are not induced by any valid instance of TH-motifs, as seen in Fig. 3 For example, in threads-ubuntu, only $0.0007 \%$ of the static h-motifs instances are induced by any valid instance of TH-motifs when $\delta$ is 5 hours. That is, DP enumerates every three connected hyperedges in $G_{T}$, ignoring any temporal information, while we are interested only in three connected temporal hyperedges that arrive within in a short period of time.

\section{B. THYME: Preliminary Version of the Proposed Algorithm}

To address the limitations of DP, we present THYME, a preliminary version of our proposed algorithm $\mathrm{THYME}^{+}$. THYME directly enumerates each instance of TH-motifs, instead of those of static h-motifs, to avoid unnecessary search. To this end, THYME concisely considers the temporal hyperedges that occur in the $\delta$-sized temporal window. In response to the arrival of a new temporal hyperedge $e_{i}$ at time $t_{i}$, the temporal window moves to $\left[t_{i}-\delta, t_{i}\right]$. It maintains only a succinct projected graph $P=\left(V_{P}, E_{P}\right)$ that represents the connectivity between the temporal hyperedges that occur within the current temporal window. As the window moves, the projected graph $P$ is incrementally updated, reflecting the changes of the current temporal hyperedges. Using $P$, THYME exhaustively enumerates the instances of TH-motifs.

Projected Graph in THYME: The projected graph $P=$ $\left(V_{P}, E_{P}\right)$ is a graph where each node is a temporal hyperedge and two nodes are connected as an edge if their corresponding temporal hyperedges share any nodes. In THYME, $P$ is maintained on the fly, with response to the temporal hyperedges 
that either enter or exit the sliding time window. The update schemes are described as insert and remove, respectively, in Algorithm 1 In insert, a temporal hyperedge $e_{i}$ is added as a node (line 14p and its neighbors (i.e., those in $V_{P}$ that are adjacent to $e_{i}$ ) are joined by edges (lines 15]16). In remove, a temporal hyperedge $e_{i}$, as well as its incident edges are removed from $V_{P}$ and $E_{P}$, respectively (lines 18 20].

Counting in THYME: The counting procedure of THYME is described in Algorithm 11. The sets of nodes and edges of the projected graph $P$ are initialized to empty maps, i.e., $V_{P}=\varnothing$ and $E_{P}=\varnothing$ (line 2). Once a temporal hyperedge $e_{i}=\left(\tilde{e}_{i}, t_{i}\right) \in \mathcal{E}$ arrives, the temporal window is moved to $\left[t_{i}-\delta, t_{i}\right]$ and the projected graph $P$ is updated accordingly, as described above. Then, it enumerates the instances of three connected nodes in $P$, which corresponds to the instances of TH-motifs of $T$ containing $e_{i}$ (line 9). For each instance $\left\langle e_{j}, e_{k}, e_{i}\right\rangle$ of TH-motif $t$, the corresponding count $M[t]$ is incremented (line 11).

Limitations of THYME: Though THYME avoids redundant search in the induced static hypergraph $G_{T}$, it directly enumerates every instance of TH-motifs in $T$. Since the size of the temporal hypergraph is much larger than that of induced static hypergraph, counting the instances in temporal hypergraph can be more computationally challenging, especially when time interval $\delta$ is large. Each temporal hyperedge within the temporal window corresponds to a unique node in the projected graph $P$ even when many temporal hyperedges are highly duplicated, as in real-world hypergraphs (see Section V-E).

\section{THYME ${ }^{+}$: Advanced Version of the Proposed Algorithm}

We present $\mathrm{THYME}^{+}$, our proposed algorithm for exactly counting the instances of TH-motifs. $\mathrm{THYME}^{+}$is faster and more efficient than DP and THYME, as shown empirically in Section $\mathrm{V}$, by addressing their limitations as follows.

- DP enumerates all instances of static h-motifs in the induced hypergraph $G_{T}$, where most of them are redundant, not induced by any instance of TH-motifs of the temporal hypergraph $T$. THYME ${ }^{+}$selectively enumerates the h-motif instances and thus reduces the redundancy.

- THYME exhaustively enumerates all instances of THmotifs. $\mathrm{THYME}^{+}$reduces the enumeration by introducing an effective counting scheme.

- The projected graph $P$ maintained by THYME can be large since each temporal hyperedge is represented as a unique node. $\mathrm{THYME}^{+}$maintains a projected graph $Q$ that is typically smaller than $P$. In $Q$, the same node can be shared by multiple temporal hyperedges. The motivation behind $Q$ is empirically demonstrated in Section V-E

Projected Graph in $\mathrm{THYME}^{+}$: $\mathrm{THYME}^{+}$maintains a projected graph $Q=\left(V_{Q}, E_{Q}, t_{Q}\right)$ composed of a set of nodes $V_{Q}$, a set of edges $E_{Q}$, and a map $t_{Q}$. Each node and edge represent a static hyperedge and a pair of static hyperedges that share any nodes, respectively. In addition, $t_{Q}$ maps a set of timestamps of temporal hyperedges inducing a particular static hyperedge. Notably, while each node in the projected

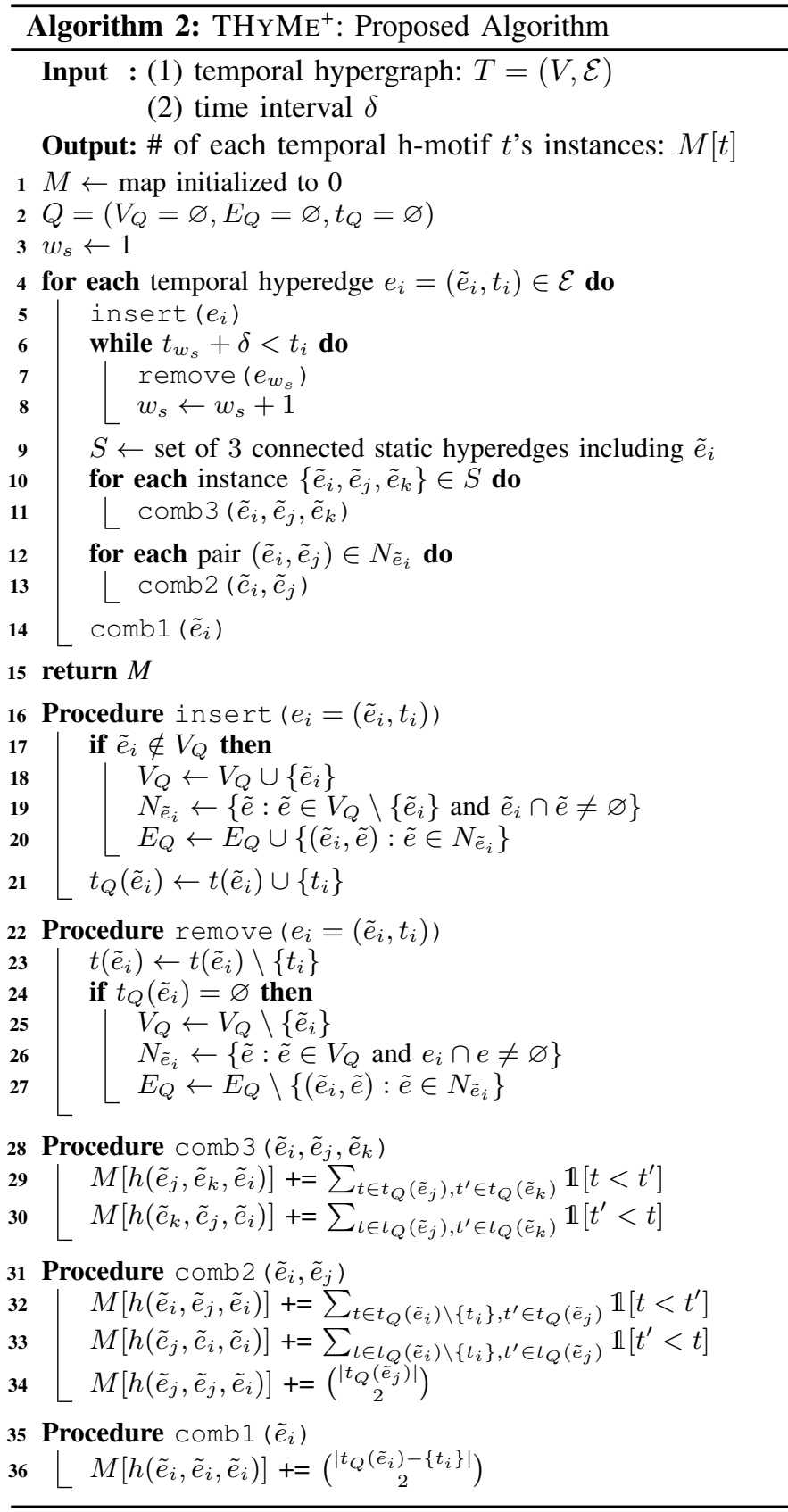

graph $P$ used in THYME is a unique temporal hyperedge, $Q$ represents the connectivity between hyperedges in the induced static hypergraph $G_{T}$. That is, duplicated temporal hyperedges can share the same node in $Q$, and thus the size of the graph can be much smaller than $P$, i.e., $\left|E_{Q}\right|<\left|E_{P}\right|$.

The update schemes of $Q$, insert and remove in Algorithm 2 add or delete nodes and their adjacent edges, respectively. More specifically, in insert, given a new temporal hyperedge $e_{i}=\left(\tilde{e}_{i}, t_{i}\right)$, its set of nodes $\tilde{e}_{i}$ is inserted as a new node, only if there do not exist any temporal hyperedges in the current temporal window whose nodes are $\tilde{e}_{i}$ (line 18). Once the new node is inserted, their incident edges are created as well (lines 19 20. Finally, the timestamp $t_{i}$ is added in $t_{Q}\left(\tilde{e}_{i}\right)$ (line 21). In remove, given a temporal hyperedge $e_{i}$ to be 
removed, it first deletes its timestamp $t_{i}$ from $t_{Q}\left(\tilde{e}_{i}\right)$ (line 23). If the $e_{i}$ is the only temporal hyperedge in the current window whose node set is $\tilde{e}_{i}$, then $\tilde{e}_{i}$ and its incident edges are removed from $V_{Q}$ and $E_{Q}$, respectively (lines 25, 27.

Counting in THYME : The counting procedure of $\mathrm{THYME}^{+}$is described in Algorithm 2. The sets of nodes and edges of the projected graph $Q$ are initialized to empty maps, i.e., $V_{Q}=\varnothing$ and $E_{Q}=\varnothing$ (line 2). For each temporal hyperedge $e_{i}=\left(\tilde{e}_{i}, t_{i}\right)$, it moves the temporal window to $\left[t_{i}-\delta, t_{i}\right]$ and accordingly as described above. Once $Q$ is updated, $\mathrm{THYME}^{+}$counts the instances of $\mathrm{TH}$-motifs that contains $e_{i}$ and the previous temporal hyperedges. To minimize enumerations, $\mathrm{THYME}^{+}$adapts effective counting schemes, comb3, comb2, and comb1, which compute the number of instances of triple-inducing, pair-inducing, and single-inducing TH-motifs, respectively, as follows:

- Triple-inducing TH-motifs (lines 9 11): $\mathrm{THYME}^{+}$first enumerates the instances of three connected hyperedges in $Q$ such that contains $\tilde{e}_{i}$ (line 9. For each set $\left\{\tilde{e}_{i}, \tilde{e}_{j}, \tilde{e}_{k}\right\}$ of three connected hyperedges, the number of instances of TH-motifs that contains $e_{i}$ is counted by timestamp combinations using comb3 method. That is, since $e_{i}$ is the latest temporal hyperedge, the set $\left\{\tilde{e}_{i}, \tilde{e}_{j}, \tilde{e}_{k}\right\}$ can be induced by sequences of either $\left\langle e_{x}, e_{y}, e_{i}\right\rangle$ or $\left\langle e_{y}, e_{x}, e_{i}\right\rangle$ where $\tilde{e}_{x}=\tilde{e}_{j}$ and $\tilde{e}_{y}=\tilde{e}_{k}$. Since $t_{x} \in t_{Q}\left(\tilde{e}_{j}\right)$ and $t_{y} \in t_{Q}\left(\tilde{e}_{k}\right)$, the number of such instances can be computed by the number of timestamp combinations of $t_{Q}\left(\tilde{e}_{j}\right)$ and $t_{Q}\left(\tilde{e}_{k}\right)$ (lines 29, 30,

- Pair-inducing TH-motifs (lines 12, 13): $\mathrm{THYME}^{+}$enumerates each edge $\left(\tilde{e}_{i}, \tilde{e}_{j}\right)$ in $Q$ that are adjacent to $\tilde{e}_{i}$, which can be induced by three different orders of sequences, $\left\langle e_{x}, e_{y}, e_{i}\right\rangle,\left\langle e_{y}, e_{x}, e_{i}\right\rangle$, and $\left\langle e_{y}, e_{y}, e_{i}\right\rangle$ where $\tilde{e}_{x}=\tilde{e}_{i}$ and $\tilde{e}_{y}=\tilde{e}_{j}$. Since $t_{x} \in t_{Q}\left(\tilde{e}_{i}\right) \backslash\left\{t_{i}\right\}$ and $t_{y} \in t_{Q}\left(\tilde{e}_{j}\right)$, the number of the sequences can be computed by the number of combinations of the set of these timestamps (lines 32 33).

- Single-inducing TH-motifs (line 14): Single-inducing THmotif, which consists of three duplicated temporal hyperedges, can be immediately counted using comb1. That is, a sequence $\left\langle e_{x}, e_{y}, e_{i}\right\rangle$ where $\tilde{e}_{x}=\tilde{e}_{i}$ and $\tilde{e}_{y}=\tilde{e}_{i}$ can be an instance of single-inducing TH-motif. Since $t_{x} \in t_{Q}\left(\tilde{e}_{i}\right) \backslash\left\{t_{i}\right\}, t_{y} \in t_{Q}\left(\tilde{e}_{i}\right) \backslash\left\{t_{i}\right\}$, and $t_{x}<t_{y}$, the number of such instances is computed immediately (line 36.

In Section V-E, we share empirical observations supporting the intuition behind $\mathrm{THYME}^{+}$. In addition, we provide the complexity analysis of $\mathrm{THYME}^{+}$in the supplementary document [29].

\section{EMPIRICAL STUDIES}

In this section, we review experiments to answer Q1-Q4.

Q1. Discoveries: Which findings do TH-motifs bring?

Q2. Comparison with Static H-motifs: Are TH-motifs more informative than static hypergraph motifs [6]?

Q3. Speed \& Efficiency: How fast and efficient is $\mathrm{THYME}^{+}$?

Q4. Further Analysis: Why is $\mathrm{THYME}^{+}$fast and efficient?
TABLE II: Statistics of the 11 real-world hypergraphs from 5 different domains: the number of nodes $|V|$, the number of temporal hyperedges $|\mathcal{E}|$, the number of induced static hyperedges $\left|E_{\mathcal{E}}\right|$, and the maximum hyperedge size $\max _{e \in \mathcal{E}}|e|$.

\begin{tabular}{c|c|c|c|c}
\hline Dataset & $|\boldsymbol{V}|$ & $|\mathcal{E}|$ & $\left|\boldsymbol{E}_{\mathcal{E}}\right|$ & $\boldsymbol{m a x}_{\boldsymbol{e} \in \mathcal{E}}|\boldsymbol{e}|$ \\
\hline email-Enron & 143 & 10,885 & 1,514 & 37 \\
email-Eu & 986 & 235,263 & 25,148 & 40 \\
\hline contact-primary & 242 & 106,879 & 12,704 & 5 \\
contact-high & 327 & 172,035 & 7,818 & 5 \\
\hline threads-ubuntu & 90,054 & 192,947 & 166,999 & 14 \\
threads-math & 153,806 & 719,792 & 595,749 & 21 \\
\hline tags-ubuntu & 3,021 & 271,233 & 147,222 & 5 \\
tags-math & 1,627 & 822,059 & 170,476 & 5 \\
\hline coauth-DBLP & $1,836,596$ & $3,700,681$ & $2,467,389$ & 280 \\
coauth-Geology & $1,091,979$ & $1,591,166$ & $1,204,704$ & 284 \\
coauth-History & 503,868 & $1,813,147$ & 896,062 & 925 \\
\hline
\end{tabular}

We first describe the settings where the experiments are conducted. Then, we provide some empirical observations using the proposed concepts and algorithms. Next, we test the scalability of the methods. Finally, we provide possible reasons why $\mathrm{THYME}^{+}$is efficient based on the observations on real-world temporal hypergraphs.

\section{A. Experimental Settings}

Machines: We conducted all the experiments on a machine with i9-10900K CPU and 64GB RAM.

Implementation: We implemented DP, THYME, and $\mathrm{THYME}^{+}$commonly in $\mathrm{C}++$.

Datasets: We use eleven real-world temporal hypergraphs from five different domains. Refer to Table I for the summarized statistics of the hypergraphs. We provide the details of each dataset in Appendix B. While we assume that timestamps of temporal hyperedges are unique, in some dataset, this may not hold. In such cases, we randomly order the temporal hyperedges whose timestamps are identical.

\section{B. Q1. Discoveries}

In this subsection, we present several observations that $\mathrm{TH}$ motifs reveal in the 11 real-world hypergraphs. TH-motifs provide a new perspective in analyzing temporal hypergraphs.

Obs 1. Real hypergraphs are not 'random': For an accurate characterization, we compare the number of instances of TH-motifs in real-world temporal hypergraphs against that in randomized ones. To this end, we randomize the real-world temporal hypergraph using HyperCL [3], a random hypergraph generator which preserves node degrees and hyperedge sizes. Once the randomized hypergraph is generated, we randomly assign the timestamps of its temporal hyperedges. In Fig. 4 we compare the distribution of the number of instances of each TH-motif in real-world temporal hypergraphs and those in randomized ones. The distributions are clearly different, and the total number of instances is greater in real-world hypergraphs than in random hypergraphs. Specifically, the total number of TH-motifs' instances in real-world hypergraphs are $6.42 \times$, $1.44 \times, 46.69 \times, 4.30 \times$ of that in randomized hypergraphs 


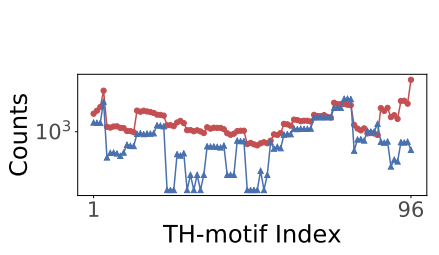

(a) email-Eu

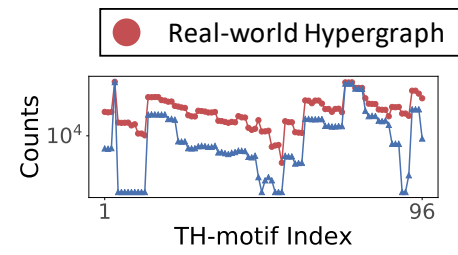

(b) contact-primary

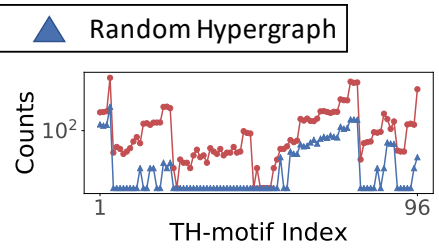

(c) threads-math

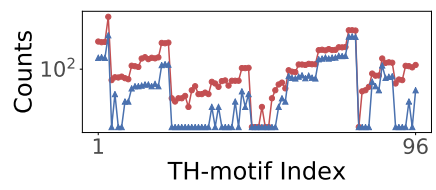

(d) tags-ubuntu

Fig. 4: The distribution of the number of TH-motifs' instances in real-world temporal hypergraphs and that in randomized temporal hypergraphs are significantly different. We set $\delta$ to 1 hour. We do not directly compare the distributions from the coauthorship datasets since their timestamp units are years. We provide the distributions in [29].

in email-Eu, contact-primary, threads-math, and tags-ubuntu, respectively.

Obs 2. TH-motifs distinguish domains: Network motifs have demonstrated their power to distinguish graphs based on their domains. In addition, the count distributions of h-motifs in static hypergraphs are particularly similar between domains but different across domains. To confirm that temporal h-motifs also possess such distinguishing power, we obtain the characteristic profile (CP) of each hypergraph, a normalized 96 dimensional vector of concatenation of relative significance of each temporal h-motif, as suggested in [6]. As seen in Fig. 5. CPs accurately capture patterns of real-world temporal hypergraphs. That is, while CPs of the temporal hypergraphs from the same domain are similar, they are different across domains. These results support that TH-motifs play a key role in capturing structural and temporal patterns of real-world temporal hypergraphs.

Obs 3. Orders of hyperedges matter: TH-motifs are asymmetric with respect to the arrival order of the temporal hyperedges, and thus instances that are indistinguishable with static h-motifs can be categorized as different TH-motifs. We are interested in how the orders of the hyperedges affect the occurrences of TH-motifs, and to this end, we statistically investigate nine pair-inducing ones, ranging from $\mathrm{TH}$-motif 87 to 95 . TH-motifs in each triple, TH-motifs $87-89$, THmotifs $90-92$, and TH-motifs $93-95$ share the same structural pattern and are distinguished by the orders of the hyperedges. Consider an instance $\left\langle e_{i}, e_{j}, e_{k}\right\rangle$ of the pair-inducing $\mathrm{TH}$ motif. The pair-inducing TH-motifs, by definition, consist of a pair of duplicated hyperedges and thus enables three different orderings 01: $\tilde{e}_{i}=\tilde{e}_{j} \neq \tilde{e}_{k}$, O2: $\tilde{e}_{i} \neq \tilde{e}_{j}=\tilde{e}_{k}$, and O3: $\tilde{e}_{i} \neq \tilde{e}_{j} \neq \tilde{e}_{k}, \tilde{e}_{i}=\tilde{e}_{k}$,. In $\mathbf{O 1}$ and $\mathbf{O 2}$, duplicated temporal hyperedges occur consecutively, whereas in $\mathbf{O 3}$, the first and last hyperedges are duplicated. TH-motifs 87, 90, and 93 are O1, TH-motifs 88, 91, and 94 are $\mathbf{O 2}$, and TH-motifs 89, 92, and 95 are $\mathbf{~ O 3}$. As seen in Fig. 6, this difference indeed affect the occurrences of the TH-motifs in real-world temporal hypergraphs. The ratio of the TH-motifs whose ordering is $\mathbf{O 3}$ are significantly small, compared to that of $\mathbf{O 1}$ and $\mathbf{O 2}$. That is, duplicated temporal hyperedges tend to occur in a short time and thus affect the count distributions of TH-motifs.

\section{Q2. Comparison with Static H-motifs}

In this subsection, we demonstrate the usefulness of $\mathrm{TH}$ motifs. We compare TH-motifs and static h-motifs as inputs features for a hyperedge prediction task.

Obs 4. TH-motifs help predict future hyperedges: To verify the usefulness of temporal h-motifs, we consider the problem of hyperedge prediction, a binary classification problem of predicting whether the given hyperedge is true or not. Given a temporal hypergraph $T=(V, \mathcal{E})$, we generate a set $\mathcal{E}^{\prime}$ of fake hyperedges, whose size is equal to the true one (i.e., $|\mathcal{E}|=\left|\mathcal{E}^{\prime}\right|$ ), using HyperCL [3], which preserves the degrees of the nodes and the sizes are equal to the true ones. The timestamps of the fake hyperedges are randomly assigned. We sort the entire temporal hyperedges $\mathcal{E} \cup \mathcal{E}^{\prime}$ based on their timestamps and split into train and test sets in a ratio 8:2. Then we train a logistic regression classifier using the train set with following three different features of each temporal hyperedge:

- THM96 $\left(\in \mathbb{R}^{96}\right)$ : Each dimension represents the number of instances of TH-motifs that contain the hyperedge.

- THM26 $\left(\in \mathbb{R}^{26}\right)$ : The 26 TH-motifs whose occurrences have the highest variance are selected.

- SHM26 $\left(\in \mathbb{R}^{26}\right)$ : Each dimension represent the number of instances of static h-motifs that contain the hyperedge. Temporal information is ignored.

As seen in Fig. 7, THM96 and THM26, which are based on the TH-motifs counts, are more accurate than STM26. While h-motifs only represent structural patterns, TH-motifs incorporate temporal information in addition to them, and thus they are more informative.

\section{Q3. Speed and Efficiency}

We evaluate the speed and efficiency of the proposed algorithms DP, THYME, and $\mathrm{THYME}^{+}$. As seen in Fig. 8 . while DP and THYME run out of memory in some datasets or with particular $\delta$ values, $\mathrm{THYME}^{+}$is fast and space efficient enough in all considered settings. Specifically, $\mathrm{THYME}^{+}$is up to $2,163 \times$ faster than DP and $16 \times$ faster than THYME. As described in Section IV] $\mathrm{THYME}^{+}$maintains a small projected graph $Q$ and thus reduces enumeration over the instances in $Q$. In the next subsection, we provide empirical findings that support the effectiveness of $\mathrm{THYME}^{+}$.

\section{E. Q4. Further Analysis}

Why is $\mathrm{THYME}^{+}$faster and more space efficient compared to DP and THYME? What properties of real-world temporal hypergraphs make $\mathrm{THYME}^{+}$efficient? To answer these questions, we examine structural and temporal patterns of temporal 

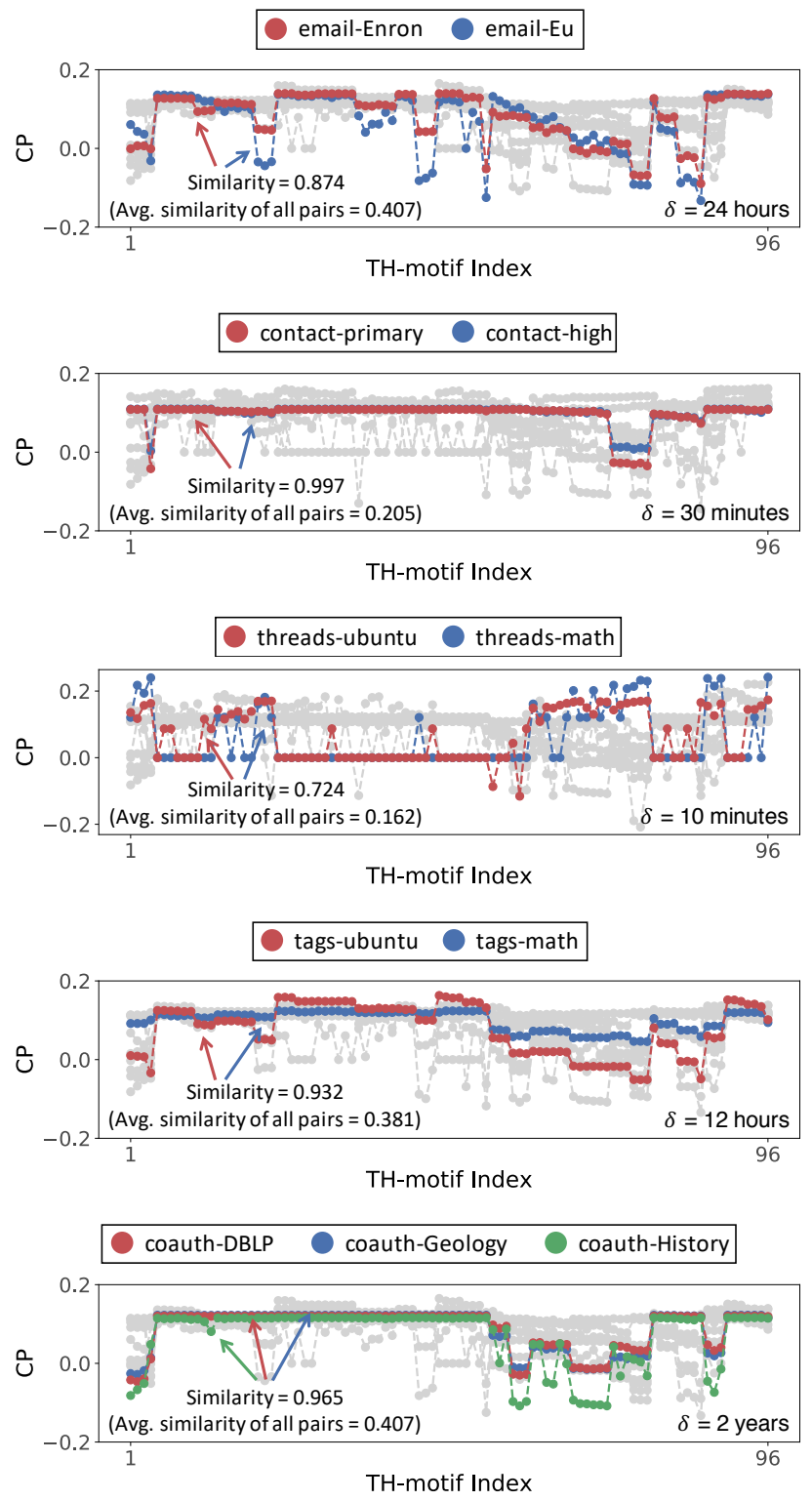

Fig. 5: Characteristic profiles (CPs) (i.e., normalized significance of each TH-motif) accurately capture the patterns of real-world temporal hypergraphs. The CPs of the temporal hypergraphs from the same domain are similar in terms of the Pearson correlation coefficients, which are the reported numbers, while they are different across domains. Grey lines indicate CPs of the temporal hypergraphs from other domains.

hyperedges in real-world temporal hypergraphs and summarize common properties observed as follows.

- (Obs. 5) Repetitive behavior: Duplicated temporal hyperedges tend to appear repeatedly, and the distribution of the numbers of repetitions is heavy-tailed.

- (Obs. 6) Temporally locality: Future temporal hyperedges are more likely to repeat recent hyperedges than older ones.

Obs. 5. Repetitive behavior: We first investigate the repeating patterns (i.e., duplication) of temporal hyperedges in real-world temporal hypergraphs. As seen in Table III, the

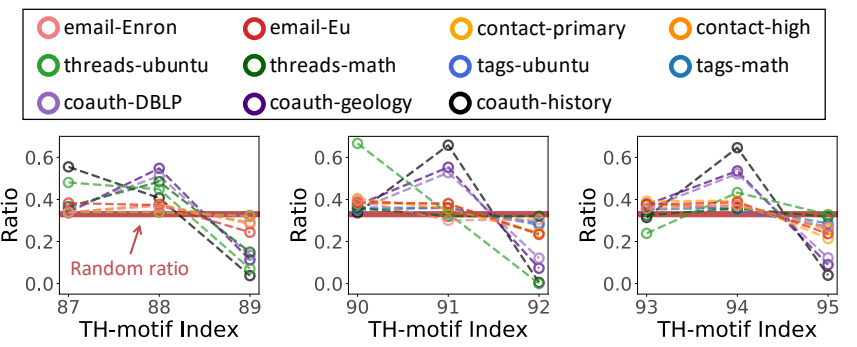

$\begin{array}{lll}\text { (a) TH-motifs 87-89 } & \text { (b) TH-motifs 90-92 } & \text { (c) TH-motifs 93-95 }\end{array}$

Fig. 6: The number of instances of nine pair-inducing THmotifs depend on the ordering of the hyperedges. The ratio of the occurrences of TH-motifs 89,92 , and 95 are significantly low compared to the other TH-motifs with same structures.

number of induced hyperedges $\left(\left|E_{\mathcal{E}}\right|\right)$ is significantly smaller than that of temporal hyperedges $(|\mathcal{E}|)$, implying that temporal hyperedges are frequently repeated. Surprisingly, in contact-high dataset, the number of induced hyperedges is only $4.5 \%$ of that of temporal hyperedges, implying that most temporal hyperedges consist of predefined set of nodes. Note that due to the flexibility of hyperedge sizes, a hyperedge can be generated from $O\left(2^{|V|}\right)$, and thus is extremely unlikely to repeat the exact set of nodes. In addition, we discover that the distributions of hyperedge repetitions in real-world temporal hypergraphs are generally heavy-tailed and close to power-law distributions, as seen in Fig. 9. We support this claim by fitting the distributions to representative heavy-tailed distributions in [29].

Obs. 6. Temporal locality: Now that we have observed the structural behaviors of the temporal hyperedges, we turn our attention to the temporal aspect. The temporal locality of temporal hyperedges is the tendency that recent hyperedges are more likely to be repeated in the near future than the older ones. To show the temporal locality, we investigate the time intervals of the $N$ consecutive identical temporal hyperedges, i.e., the time it takes for a hyperedge to be repeated $N$ times. Fig. 10 shows the average time intervals of all the hyperedges in the real-world hypergraphs and randomly shuffled hypergraphs, where timestamps of the hyperedges are randomly shuffled while preserving the underlying structure. In every dataset, the time intervals within $N$ consecutive hyperedges are shorter in real-world hypergraphs than in randomized ones. That is, future hyperedges are more likely to repeat the recent hyperedges than older ones.

Intuition behind THYME ${ }^{+}$: How do these properties of realworld temporal hypergraphs provide efficiency to $\mathrm{THYME}^{+}$? Here, we provide some reasons why we expect $\mathrm{THYME}^{+}$to be faster and more space-efficient than THYME and DP.

- Connection to Obs. 5: Each node in the projected graph $P$ used in THYME represents a unique temporal hyperedge, and its size heavily depends on $\delta$. On the other hand, the nodes in the projected graph $Q$ maintained in $\mathrm{THYME}^{+}$ represent induced hyperedges, and several temporal hyperedges can share the same node. Thus, more repetitions of temporal hyperedges provide higher efficiency of $\mathrm{THYME}^{+}$, 


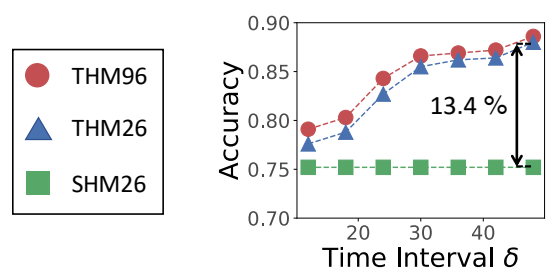

(a) email-Enron

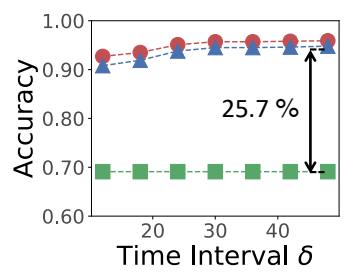

(b) email-Eu

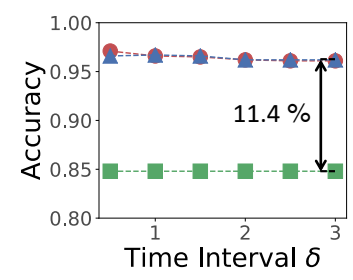

(c) contact-primary

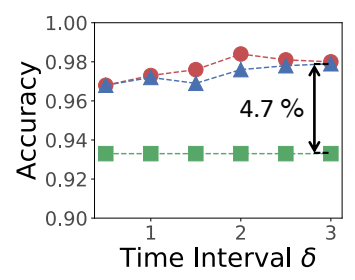

(d) contact-high

Fig. 7: TH-motifs provide informative features of temporal hyperedges. THM96 and THM26, which use the counts of THmotifs' instance as features, are more accurate than SHM26, which uses the counts of static h-motifs' instances, in predicting future temporal hyperedges. Results in small datasets where the instances of static h-motifs can be exactly counted are reported.

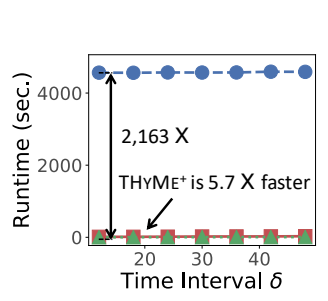

(a) email-Eu

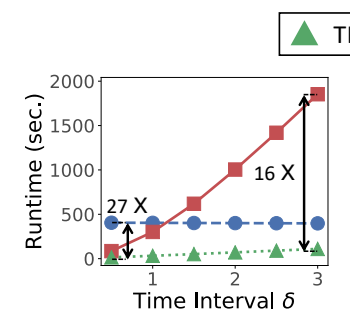

(b) contact-primary

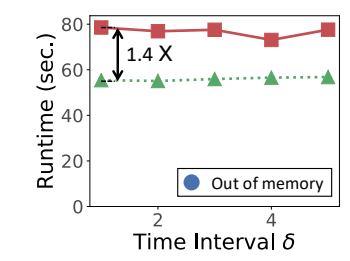

(c) threads-math

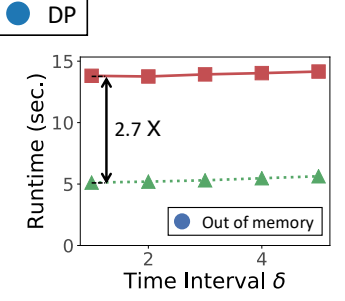

(d) tags-ubuntu

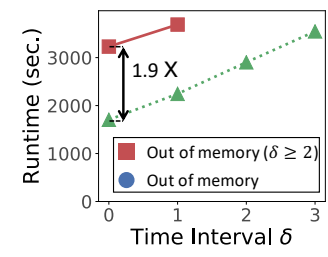

(e) coauth-DBLP

Fig. 8: $\mathrm{THYME}^{+}$is faster and more space efficient than DP and THYME. We provide the full results in [29].

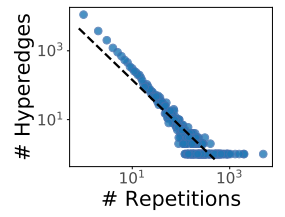

(a) email-Eu

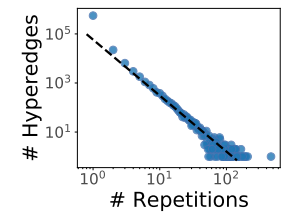

(b) threads-math

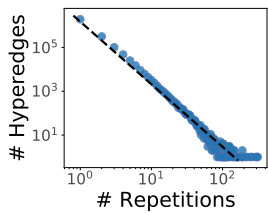

(c) coauth-DBLP

Fig. 9: Temporal hyperedges in real-world hypergraphs are repetitive. Temporal hyperedges appear repetitively and the number of repetitions follow a near power-law distribution. This tendency is found consistently across all datasets [29].

as observed in real-world temporal hypergraphs.

- Connection to Obs. 6: The benefits of temporal locality of temporal hyperedges are two-fold: (1) The tendency of temporal hyperedges to repeat within a short period of time indicates that duplicated temporal hyperedges are more likely to co-appear in the temporal window in $\mathrm{THYME}^{+}$, which reduces the size of the projected graph $Q$. (2) If duplicated temporal hyperedges reappear within the temporal window, insertion/deletion of nodes and edges of $Q$ are skipped, which is beneficial in terms of speed.

\section{CONCLUSION}

In this work, we propose (a) temporal hypergraph motifs (TH-motifs), which are tools for analyzing design principles of time-evolving hypergraphs, and (b) $\mathrm{THYME}^{+}$, which is a fast algorithm for exactly counting TH-motifs' instances. Using them, we investigate 11 real-world hypergraphs from 5 domains. Our contributions are summarized as follows.

- New concept: We define 96 temporal hypergraph motifs (TH-motifs) that describe local relational and temporal dynamics in time-evolving hypergraphs.

- Fast and exact algorithm: We develop $\mathrm{THYME}^{+}$, a fast and exact algorithm for counting the instances of TH-motifs. It is at most $2,163 \times$ faster than the baseline approach.

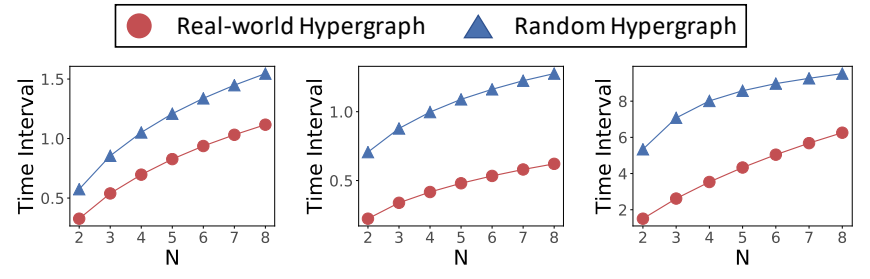
(a) email-Eu
(b) threads-math
(c) coauth-DBLP

Fig. 10: Temporal hyperedges in real-world hypergraphs are temporally local. The time intervals of $N$ consecutive duplicated temporal hyperedges is shorter in real-world temporal hypergraphs than in randomized hypergraphs. The units of time intervals in coauth-DBLP is years, and the others are hours. We provide the full results in [29].

- Empirical discoveries: TH-motifs reveal interesting structural and temporal patterns in real-world hypergraphs. THmotifs also provide informative features that are useful in predicting future hyperedges.

Reproducibility: The source code and datasets used in this work are available at https://github.com/geonlee0325/THyMe

Acknowledgements: This work was supported by National Research Foundation of Korea (NRF) grant funded by the Korea government (MSIT) (No. NRF-2020R1C1C1008296) and Institute of Information \& Communications Technology Planning \& Evaluation (IITP) grant funded by the Korea government (MSIT) (No. 2019-0-00075, Artificial Intelligence Graduate School Program (KAIST)).

\section{REFERENCES}

[1] Y. Kook, J. Ko, and K. Shin, "Evolution of real-world hypergraphs: Patterns and models without oracles," in ICDM, 2020.

[2] M. T. Do, S.-e. Yoon, B. Hooi, and K. Shin, "Structural patterns and generative models of real-world hypergraphs," in $K D D, 2020$.

[3] G. Lee, M. Choe, and K. Shin, "How do hyperedges overlap in realworld hypergraphs?-patterns, measures, and generators," in $W W W, 2021$.

[4] A. R. Benson, R. Kumar, and A. Tomkins, "Sequences of sets," in $K D D$, 2018. 
[5] A. R. Benson, R. Abebe, M. T. Schaub, A. Jadbabaie, and J. Kleinberg, "Simplicial closure and higher-order link prediction," Proceedings of the National Academy of Sciences, vol. 115, no. 48, pp. E11221-E11230, 2018.

[6] G. Lee, J. Ko, and K. Shin, "Hypergraph motifs: concepts, algorithms, and discoveries," PVLDB, vol. 13, pp. 2256-2269, 2020.

[7] R. Milo, S. Shen-Orr, S. Itzkovitz, N. Kashtan, D. Chklovskii, and U. Alon, "Network motifs: simple building blocks of complex networks," Science, vol. 298, no. 5594, pp. 824-827, 2002.

[8] S.-e. Yoon, H. Song, K. Shin, and Y. Yi, "How much and when do we need higher-order information in hypergraphs? a case study on hyperedge prediction," in $W W W, 2020$.

[9] A. Paranjape, A. R. Benson, and J. Leskovec, "Motifs in temporal networks," in WSDM, 2017.

[10] Y. Li, Z. Lou, Y. Shi, and J. Han, "Temporal motifs in heterogeneous information networks," in MLG Workshop, 2018.

[11] L. Kovanen, M. Karsai, K. Kaski, J. Kertész, and J. Saramäki, “Temporal motifs in time-dependent networks," Journal of Statistical Mechanics: Theory and Experiment, vol. 2011, no. 11, p. P11005, 2011.

[12] S. Gurukar, S. Ranu, and B. Ravindran, "Commit: A scalable approach to mining communication motifs from dynamic networks," in SIGMOD, 2015.

[13] U. Redmond and P. Cunningham, "Temporal subgraph isomorphism," in ASONAM, 2013.

[14] S. S. Shen-Orr, R. Milo, S. Mangan, and U. Alon, "Network motifs in the transcriptional regulation network of escherichia coli," Nature Genetics, vol. 31, no. 1, pp. 64-68, 2002.

[15] R. Milo, S. Itzkovitz, N. Kashtan, R. Levitt, S. Shen-Orr, I. Ayzenshtat, M. Sheffer, and U. Alon, "Superfamilies of evolved and designed networks," Science, vol. 303, no. 5663, pp. 1538-1542, 2004.

[16] R. A. Rossi, N. K. Ahmed, A. Carranza, D. Arbour, A. Rao, S. Kim, and E. Koh, "Heterogeneous graphlets," ACM TKDD, vol. 15, no. 1, pp. $1-43,2020$.

[17] S. P. Borgatti and M. G. Everett, "Network analysis of 2-mode data," Social networks, vol. 19, no. 3, pp. 243-269, 1997.

[18] A. R. Benson, D. F. Gleich, and J. Leskovec, "Higher-order organization of complex networks," Science, vol. 353, no. 6295, pp. 163-166, 2016.

[19] P.-Z. Li, L. Huang, C.-D. Wang, and J.-H. Lai, "Edmot: An edge enhancement approach for motif-aware community detection," in $K D D$, 2019.

[20] C. E. Tsourakakis, J. Pachocki, and M. Mitzenmacher, "Scalable motifaware graph clustering," in $W W W, 2017$.

[21] H. Yin, A. R. Benson, J. Leskovec, and D. F. Gleich, "Local higher-order graph clustering," in KDD, 2017.

[22] A. Arenas, A. Fernandez, S. Fortunato, and S. Gomez, "Motif-based communities in complex networks," Journal of Physics A: Math Theor, vol. 41, no. 22, p. 224001, 2008.

[23] H. Zhao, X. Xu, Y. Song, D. L. Lee, Z. Chen, and H. Gao, "Ranking users in social networks with higher-order structures," in $A A A I, 2018$.

[24] Y. Yu, Z. Lu, J. Liu, G. Zhao, and J.-r. Wen, "Rum: Network representation learning using motifs," in ICDE, 2019.

[25] R. A. Rossi, N. K. Ahmed, and E. Koh, "Higher-order network representation learning," in WWW Companion, 2018.

[26] R. A. Rossi, N. K. Ahmed, E. Koh, S. Kim, A. Rao, and Y. AbbasiYadkori, "A structural graph representation learning framework," in WSDM, 2020.

[27] J. B. Lee, R. A. Rossi, X. Kong, S. Kim, E. Koh, and A. Rao, "Graph convolutional networks with motif-based attention," in CIKM, 2019.

[28] R. A. Rossi, R. Zhou, and N. K. Ahmed, "Deep inductive graph representation learning," IEEE TKDE, vol. 32, no. 3, pp. 438-452, 2018.

[29] "Online appendix," 2021. [Online]. Available: https://github.com/ geonlee0325/THyMe/blob/main/supplements.pdf

\section{APPENDIX}

\section{A. Details of Dynamic Programming (DP)}

The procedure count (lines 9019 counts the instances of TH-motifs that induce a set of $\ell$ connected static hyperedges. That is, given a set $s=\left\{\tilde{e}_{1}, \ldots, \tilde{e}_{\ell}\right\}$ of $\ell$ connected static hyperedges, count first constructs a time-sorted sequence $e(s)$ of temporal hyperedges whose nodes is one of $s$ (line 10 . It also introduces a map $C$ that maintains the counts of ordered hyperedges of length at most $\ell$. Then count scans through the temporal hyperedges in $e(s)$ and tracks the subsequences that occur within the temporal window that spans temporal hyperedges within $\delta$ time units. As the temporal window slides through the temporal hyperedges $e(s)$, the count of the sequences are computed based on the subsequences counted in $C$. Refer to [9] for more intuition behind this dynamic programming formulation.

\begin{tabular}{l}
\hline Algorithm 3: DP: Preliminary Algorithm for Exact \\
Counting of TH-motifs' Instances
\end{tabular}

Input : (1) temporal hypergraph: $T=(V, \mathcal{E})$

(2) time interval $\delta$

Output: \# of each temporal h-motif $t$ 's instances: $M[t]$

$1 S \leftarrow$ set of instances of static h-motifs in $G_{T}$

2 for each instance $\left\{\tilde{e}_{i}, \tilde{e}_{j}, \tilde{e}_{k}\right\} \in S$ do

$3\left\lfloor\operatorname{count}\left(\left\{\tilde{e}_{i}, \tilde{e}_{j}, \tilde{e}_{k}\right\}\right)\right.$

4 for each pair of overlapping hyperedges $\left\{\tilde{e}_{i}, \tilde{e}_{j}\right\} \in \wedge_{\mathcal{E}}$ do

$5\left\lfloor\operatorname{count}\left(\left\{\tilde{e}_{i}, \tilde{e}_{j}\right\}\right)\right.$

6 for each hyperedge $\tilde{e}_{i} \in E_{\mathcal{E}}$ do

$7\left\lfloor\operatorname{count}\left(\left\{\tilde{e}_{i}\right\}\right)\right.$

8 return $M$

9 Procedure count $\left(s=\left\{\tilde{e}_{1}, \ldots, \tilde{e}_{\ell}\right\}\right)$

$10 \quad e(s) \leftarrow \operatorname{sorted}\left(I\left(\tilde{e}_{1}\right) \cup \cdots \cup I\left(\tilde{e}_{\ell}\right)\right)$

$11 \quad w_{s} \leftarrow 1$

$12 \quad C \leftarrow$ map initialized to 0

13 for each temporal hyperedge $e_{i}=\left(\tilde{e}_{i}, t_{i}\right) \in e(s)$ do

14 while $t_{w_{s}}+\delta<t_{i}$ do

$15 \quad$ decrement $\left(\tilde{e}_{w_{s}}\right)$

$16 \quad\left[w_{s} \leftarrow w_{s}+1\right.$

$17 \quad$ increment $\left(\tilde{e}_{i}\right)$

18 for each $\left\langle e_{i}, e_{j}, e_{k}\right\rangle \in \operatorname{permutations}\left(\left\{\tilde{e}_{i}, \tilde{e}_{j}, \tilde{e}_{k}\right\}\right)$ do

$19 \quad\left\lfloor M\left[h\left(\tilde{e}_{i}, \tilde{e}_{j}, \tilde{e}_{k}\right)\right]+=C\left[\operatorname{concat}\left(\tilde{e}_{i}, \tilde{e}_{j}, \tilde{e}_{k}\right)\right]\right.$

20 Procedure increment ( $\tilde{e})$

21 for each prefix in $C$.keys.reverse of length $<\ell$ do

$22 \quad\lfloor C[\operatorname{concat}(\operatorname{prefix}, \tilde{e})]+=C[$ prefix $]$

$23 \quad C[\tilde{e}] \leftarrow C[\tilde{e}]+1$

24 Procedure decrement ( $\tilde{e})$

$25 \mid C[\tilde{e}] \leftarrow C[\tilde{e}]-1$

26 for each suffix in $C$.keys of length $<\ell-1$ do

$27 \quad\lfloor C[\operatorname{concat}(\tilde{e}, \operatorname{suffix})]-=C[\operatorname{suffix}]$

\section{B. Details of Datasets}

We provide the details of the eleven real-world temporal hypergraphs from the following five distinct domains:

- email: Each node is an email account and each hyperedge is the set of sender and receivers of the email.

- contact: Each node is a person and each hyperedge is a group interaction among people.

- threads: Each node is a user and each hyperedge is a group of users working in a thread.

- tags: Each node is a tag and each hyperedge is a set of tags attached to the question.

- coauthorship: Each node is an author and each hyperedge is a set of authors of the publication. 